\title{
Notch Signaling Influences Neuroprotective and Proliferative Properties of Mature Müller Glia
}

\author{
Kanika Ghai, ${ }^{1,2}$ Christopher Zelinka, ${ }^{1}$ and Andy J. Fischer ${ }^{1}$ \\ 'Department of Neuroscience and ${ }^{2}$ Neurosciences Graduate Studies Program, The Ohio State University, Columbus, Ohio 43210
}

\begin{abstract}
Notch signaling is known to play important roles during retinal development. Recently, Notch signaling has been shown to be active in proliferating Müller glia in acutely damaged chick retina (Hayes et al., 2007). However, the roles of Notch in mature, undamaged retina remain unknown. Thus, the purpose of this study was to determine the role of the Notch-signaling pathway in the postnatal retina. Here we show that components of the Notch-signaling pathway are expressed in most Müller glia at low levels in undamaged retina. The expression of Notch-related genes varies during early postnatal development and across regions, with higher expression in peripheral versus central retina. Blockade of Notch activity with a small molecule inhibitor before damage was protective to retinal interneurons (amacrine and bipolar cells) and projection neurons (ganglion cells). In the absence of damage, Notch is upregulated in retinas treated with insulin and FGF2; the combination of these factors is known to stimulate the proliferation and dedifferentiation of Müller glia (Fischer et al., 2002b). Inhibition of Notch signaling during FGF2 treatment reduces levels of the downstream effectors of the MAPKsignaling pathway-p38 MAPK and pCREB in Müller glia. Further, inhibition of Notch activity potently inhibits FGF2-induced proliferation of Müller glia. Together, our data indicate that Notch signaling is downstream of, and is required for, FGF2/MAPK signaling to drive the proliferation of Müller glia. In addition, our data suggest that low levels of Notch signaling in Müller glia diminish the neuroprotective activities of these glial cells.
\end{abstract}

\section{Introduction}

The Notch pathway is a highly conserved cell-signaling system that regulates many aspects of embryonic development (Louvi and Artavanis-Tsakonas, 2006). Notch signaling maintains progenitor cells in an undifferentiated state during neurogenesis (Dorsky et al., 1997; Henrique et al., 1997; Rapaport and Dorsky, 1998; Gaiano and Fishell, 2002), promotes glial cell fates during retinal histogenesis (Furukawa et al., 2000; Gaiano et al., 2000; Vetter and Moore, 2001; Gaiano and Fishell, 2002), and promotes cell survival in the nervous system (Oishi et al., 2004; Mason et al., 2006; Breunig et al., 2007). Notch encodes a transmembrane receptor that binds to transmembrane ligands (Delta, Serrate, or Jagged) on the surface of adjacent cells. Receptorligand binding initiates the $\gamma$-secretase-dependent cleavage of the Notch intracellular domain, which is translocated to the nucleus and binds to suppressor-of-hairless, which upregulates a class of basic helix-loop-helix transcriptional repressors, hairy and en-

\footnotetext{
Received Oct. 2, 2009; revised Jan. 4, 2010; accepted Jan. 19, 2010.

This work was supported by a grant (EY016043) from the National Institutes of Health. We thank Drs. Heithem El-Hodiri, Susan Cole, and Jennifer Stanke for comments that contributed to the final version of this manuscript. Dr. Paul Linser provided antibodies to 2M6. Dr. Domingos Henrique provided the cNotch1 plasmid and the cHes1 (cHairy-2) sequence. Confocal microscopy was performed at the Hunt-Curtis Imaging Facility at the Department of Neuroscience of The Ohio State University. The BrdU, vimentin, and transitin antibodies developed by Drs. S. J. Kaufman and G. Cole were obtained from the Developmental Studies Hybridoma Bank under auspices of the National Institute of Child Health and Human Development and maintained by the Department of Biological Sciences, University of lowa (lowa City, IA).

Correspondence should be addressed to Andy J. Fischer, Department of Neuroscience, The Ohio State University, College of Medicine, 3020 Graves Hall, 333 West 10th Avenue, Columbus, OH 43210-1239. E-mail: fischer.412@ osu.edu.

DOI:10.1523/JNEUROSCI.4919-09.2010

Copyright $\odot 2010$ the authors $\quad 0270-6474 / 10 / 303101-12 \$ 15.00 / 0$
}

hancer of split (HES) genes. Although the mechanisms of Notch signaling are highly conserved across species, the functions of the signaling differ with the cellular context and stage of development.

The Notch pathway has been shown to play a role in retinal regeneration in lower vertebrates such as fish (Sullivan et al., 1997; Yurco and Cameron, 2005) and newts (Nakamura and Chiba, 2007). In response to excitotoxic injury, Müller glia in the chick retina dedifferentiate, proliferate, and generate a few neurons (Fischer and Reh, 2001). During this regenerative process, Müller glia have been shown to upregulate Notch signaling, which is necessary for glial dedifferentiation and proliferation (Hayes et al., 2007). Thereafter, Notch signaling inhibits the neuronal differentiation of newly generated cells (Hayes et al., 2007). Surprisingly, the role of Notch signaling in normal Müller glia in undamaged retina remains unknown.

We have previously shown that the mitogen-activated protein kinase (MAPK) pathway stimulates proliferation and transdifferentiation of Müller glia (Fischer et al., 2009a,b). This pathway is activated by secreted factors such as insulin, insulin-like growth factors (IGFs), and fibroblast growth factors (FGFs) (Grewal et al., 1999). In the absence of damage, daily injections of the combination of insulin and FGF2, but not either factor alone, stimulate Müller glia to proliferate, transdifferentiate, and produce some neurons (Fischer et al., 2002b). Intraocular injections of FGF2 activate MAPK signaling in the retina, manifested as accumulations of pERK1/2, pCREB, cFos, Egr1, and p38 MAPK in Müller glia (Fischer et al., 2009a). Further, small molecule inhibitors to ERK1/2 and the FGF receptor suppress the proliferation of Müller glia in NMDA-damaged retinas (Fischer et al., 2009a). 
Thus, active MAPK signaling is required in damaged retinas, and may be sufficient to drive the proliferation and transdifferentiation of Müller glia in undamaged retinas.

Although Notch and MAPK pathways are known to be important during glia-mediated retinal regeneration, interactions between these pathways remain unknown. Here we investigate the expression patterns and roles of the Notch pathway components in the postnatal chicken retina. Further, we investigate the interaction of the Notch pathway with the MAPK pathway in regulating Müller glial proliferation and transdifferentiation.

\section{Materials and Methods}

Animals. The use of animals was in accordance with the guidelines established by the National Institutes of Health and the Ohio State University. Newly hatched leghorn chickens (Gallus gallus domesticus) were obtained from the Department of Animal Sciences at the Ohio State University and kept on a cycle of $12 \mathrm{~h}$ light, $12 \mathrm{~h}$ dark (lights on at 7:00 A.M.). Chicks were housed in a stainless steel brooder at $\sim 28^{\circ} \mathrm{C}$ and received water and Purina chick starter ad libitum.

Intraocular injections. Intraocular injections were performed as described previously (Fischer et al., 1998, 1999). In all experiments, $20 \mu \mathrm{l}$ of vehicle containing the test compound was injected into the experimental (right) eye, and $20 \mu \mathrm{l}$ of vehicle alone was injected into the control (left) eye. The vehicle was sterile saline $\left(0.9 \% \mathrm{NaCl}\right.$ in $\left.\mathrm{dH}_{2} \mathrm{O}\right)$ containing bovine serum albumin, $50 \mu \mathrm{g} / \mathrm{ml}$, as carrier for NMDA, colchicine, FGF2, and insulin, and 50\% DMSO in sterile saline/BSA for DAPT. Test compounds included NMDA (150 or $300 \mu \mathrm{g} /$ dose; Sigma-Aldrich), colchicine (500 ng/dose; Sigma-Aldrich), recombinant human FGF2 (250 ng/ dose; R\&D Systems), purified bovine insulin (1 $\mu \mathrm{g} / \mathrm{dose}$; SigmaAldrich), DAPT ( $N$-[N-(3,5-difluorophenacetyl-L-alanyl)]-S-phenylglycine $t$-butyl ester) (865 ng/dose; Sigma Aldrich).

We used the following injection paradigms: (1) On posthatch day 5 (P5), the right eye received a single injection of DAPT, and the left eye received vehicle. Retinas were harvested $24 \mathrm{~h}$ later. (2) On P5 and P6, the right eye received an injection of DAPT, and the right eye received NMDA or colchicine. Retinas were harvested 1, 2, or 10 d later. (3) On P4, $\mathrm{P} 5$, and P6, the right eye received an injection of $1 \mu \mathrm{g}$ of insulin and 250 ng of FGF2, and the left eye received vehicle. On P7, both eyes received an injection of $2 \mu \mathrm{g}$ of BrdU, and retinas were harvested 24 or $48 \mathrm{~h}$ later. (4) On P5 and P6, the right eye received an injection of $250 \mathrm{ng}$ of FGF2, and the left eye received vehicle. Retinas were harvested 24 h later. (5) On P4, both eyes received $150 \mu \mathrm{g}$ of NMDA. On P5, the right eye received $250 \mathrm{ng}$ of FGF2, and the left eye received vehicle. On P6, the right eye received 250 ng of FGF2 and $2 \mu \mathrm{g}$ of BrdU, and the left eye received $2 \mu \mathrm{g}$ of BrdU alone. On P7, retinas were harvested $24 \mathrm{~h}$ later. (6) On P4, both eyes received $150 \mu \mathrm{g}$ of NMDA. On P5, the right eye received $250 \mathrm{ng}$ of FGF $2+865 \mathrm{ng}$ of DAPT, and the left eye received $250 \mathrm{ng}$ of FGF2 alone. On P6, the right eye received $250 \mathrm{ng}$ of FGF2 $+865 \mathrm{ng}$ of DAPT $+2 \mu \mathrm{g}$ of BrdU, and the left eye received $250 \mathrm{ng}$ of FGF2 $+2 \mu$ g of BrdU. On P7, retinas were harvested 24 h later. (7) On P4 and P5, the right eye received $250 \mathrm{ng}$ of FGF2 $+865 \mathrm{ng}$ of DAPT, and the left eye received $250 \mathrm{ng}$ of FGF2 alone. On P6, both eyes received $150 \mu \mathrm{g}$ of NMDA. On P7, both eyes received $2 \mu \mathrm{g}$ of $\mathrm{BrdU}$, and retinas were harvested $24 \mathrm{~h}$ later.

Reverse transcriptase PCR. After removing the eye from the orbit, a blade was used to cut away the posterior portion of the eye containing the central retina ( $\sim 6 \mathrm{~mm}$ diameter) from the anterior portion containing the peripheral retina (approximately within $5 \mathrm{~mm}$ of the circumferential marginal zone). mRNA was harvested from central and peripheral regions of the retinas dissected in cold HBSS by using the RNeasy Kit (Qiagen) as per the manufacturer's instructions. Purified RNA was resuspended in $100 \mu \mathrm{l}$ of RNase-free water and DNA removed by using DNase I (Ambion). cDNA was synthesized from mRNA by using oligo dT primers and Superscript III First Strand Synthesis System (Invitrogen) according to the manufacturer's protocol. Control reactions were performed by excluding the reverse transcriptase to assess whether primers were amplifying genomic DNA.

PCR primers were designed by using the web-based program Primer3 from the Whitehead Institute for Biomedical Research (http://frodo.wi. mit.edu/). Primer sequences and amplified DNA sequence lengths are
Table 1. Primer sets used for standard and real-time PCR

\begin{tabular}{|c|c|c|c|}
\hline Gene & Forward primer & Reverse primer & $\begin{array}{l}\text { Sequenc } \\
\text { length }\end{array}$ \\
\hline \multicolumn{4}{|l|}{ For standard PCR } \\
\hline cNotch1 & AAG TTC AGG TTC GAG GAG CA & GCG CCT TGG TAG ATG AAA TC & 289 \\
\hline cHes1 & GAG ATG ACC GTC AAG CACCT & TGG TAC AAA GGC ACA ATC CA & 628 \\
\hline cHes5 & CCA GAG ACA CCA ACC CAA CT & GCT GGT GAG TGG AAG TGG AT & 277 \\
\hline \multicolumn{4}{|c|}{ For real-time $P C R$} \\
\hline cNotch1 & GGC TGG TTA TCA TGG AGT TA & CAT CCA CAT TGA TCT CACAG & 154 \\
\hline cDelta1 (cDII1) & CAC TGA CAA CCC TGA TGG TG & TGG CAC TGG CAT ATG TAG GA & 152 \\
\hline CDII4 & GGT CTG CAG CGA GAA CTA CT & TGC AGT ATC CAT TCT GTT CG & 181 \\
\hline $\begin{array}{l}\text { cJag1 } \\
\quad \text { (cSerrate1) }\end{array}$ & TGA TAA GTG CAT TCC ACA CC & CAG GTA CCA CCA TTC AAA CA & 149 \\
\hline cHes1 (cHairy2) & CGC TGA AGA AGG ATA GTT CG & GTC ACT TCG TTC ATG CAC TC & 175 \\
\hline ches 5 & GGA GAA GGA GTT CCA GAG AC & AAT TGC AGA GCT TCT TTG AG & 143 \\
\hline cHey1 & TGA CAA ATC AGA TGG ACC AG & CCA TTC TGA AGT CTG CAC TG & 189 \\
\hline cHey2 & AAT GAC GGT GGA TCA TCT G & TAC CTC GCA ACT TCA GTC AA & 126 \\
\hline ascl1a & AGG GAA CCA CGT TTA TGC AG & TTA TAC AGG GCC TGG TGA GC & 187 \\
\hline FGFR1 & TGA AAC ACA TCG AGG TGA AC & ATA GAA TTA CCC GCC AAA CA & 171 \\
\hline FGFR3 & САT TCT CAG CTA TGG CAC TG & CAC CTG CTG TCT CTT GAG TG & 147 \\
\hline GAPDH & CAT CCA AGG AGT GAG CCA AG & TGG AGG AAG AAA TTG GAG GA & 134 \\
\hline
\end{tabular}

The table lists $19-20$ bp forward and reverse primer sets for the appropriate gene, along with the expected PCR product size for each primer set.

presented in Table 1. Standard PCRs were performed, in which cDNA was amplified for 20 cycles using standard protocols, Platinum Taq (Invitrogen) or TITANIUM Taq (Clontech), and an Eppendorf thermal cycler. The standard PCR products were run on an agarose gel and stained with ethidium bromide to determine the predicted product sizes.

Real-time PCR. cDNA was synthesized as described using standard protocols as described before (Fischer et al., 2004b). mRNA was harvested from retinas, reverse transcribed to generate cDNA, and amplified using real-time PCR for 40 cycles. Real-time PCR was performed using the StepOnePlus Real-Time PCR System (Applied Biosystems) according to the manufacturer's instructions. Reactions were performed in triplicates, in $25 \mu \mathrm{l}$ volumes with $0.5 \mu \mathrm{M}$ primers and $\mathrm{MgCl}_{2}$ concentration optimized between 2 and $5 \mathrm{~mm}$. Nucleotides, TaqDNA polymerase, and buffer were included in the SYBR Green PCR Master Mix (Applied Biosystems). A typical protocol designed on StepOne Software v2.0 (Applied Biosystems) included a holding stage for $10 \mathrm{~min}$ at $95^{\circ} \mathrm{C}$ and then a $15 \mathrm{~s}$ denaturation step, followed by 40 cycles with a $95^{\circ} \mathrm{C}$ denaturation for $15 \mathrm{~s}$ and $60^{\circ} \mathrm{C}$ annealing for $1 \mathrm{~min}$. The melt curve stage included $95^{\circ} \mathrm{C}$ for $15 \mathrm{~s}, 60^{\circ} \mathrm{C}$ annealing for $1 \mathrm{~min}$, and $95^{\circ} \mathrm{C}$ for $15 \mathrm{~s}$. Measurements of the fluorescence were performed at the end of the $60^{\circ} \mathrm{C}$ annealing period. Ct values obtained from real-time PCR were normalized to GAPDH, and the fold difference between control and treated samples was determined using the $\Delta \mathrm{Ct}$ method and represented as a percentage change from baseline.

In situ hybridization. A partial length clone of cNotch1 (1043 bp) was kindly provided by Dr. Domingos Henrique (Instituto de Medicina Molecular, Lisboa, Portugal). Riboprobes were synthesized using a kit provided by Roche and stored at $-80^{\circ} \mathrm{C}$ until use. Ocular tissues from P7 eyes were dissected in RNase-free HBSS (Invitrogen), fixed overnight at $4^{\circ} \mathrm{C}$ in $4 \%$ paraformaldehyde buffered in $0.1 \mathrm{M}$ dibasic sodium phosphate, and embedded in OCT compound (Tissue-Tek). Cryosections were processed for in situ hybridization with digoxigenin-labeled RNA probes as described previously (Fischer et al., 2002a, 2004b). Hybridization was detected by using Fab fragments to digoxigenin that were conjugated to alkaline phosphatase (anti-DIG-AP; Roche) diluted in MABT ( $0.05 \mathrm{M}$ maleic acid buffer, $0.1 \%$ Tween 20$)$ plus $10 \%$ normal goat serum, $10 \mathrm{~mm}$ levamisole, and $10 \mathrm{~mm}$ glycine. Nitro-blue tetrazolium (NBT) and 5-bromo-4-chloro-3'-indolyl phosphate $p$-toluidine (BCIP) in $0.1 \mathrm{M}$ $\mathrm{NaCl}, 0.1 \mathrm{M}$ Tris- $\mathrm{HCl}, \mathrm{pH}$ 9.5, $0.05 \mathrm{M} \mathrm{MgCl}_{2}$, and $0.01 \%$ Tween 20 were used to precipitate chromophore from the anti-DIG-AP. With sufficient levels of chromophore developed, the sections were washed in PBS, fixed in $4 \%$ paraformaldehyde in PBS for $15 \mathrm{~min}$, washed again in PBS, and processed for immunolabeling for $2 \mathrm{M} 6$, BrdU, and PCNA as described below. 


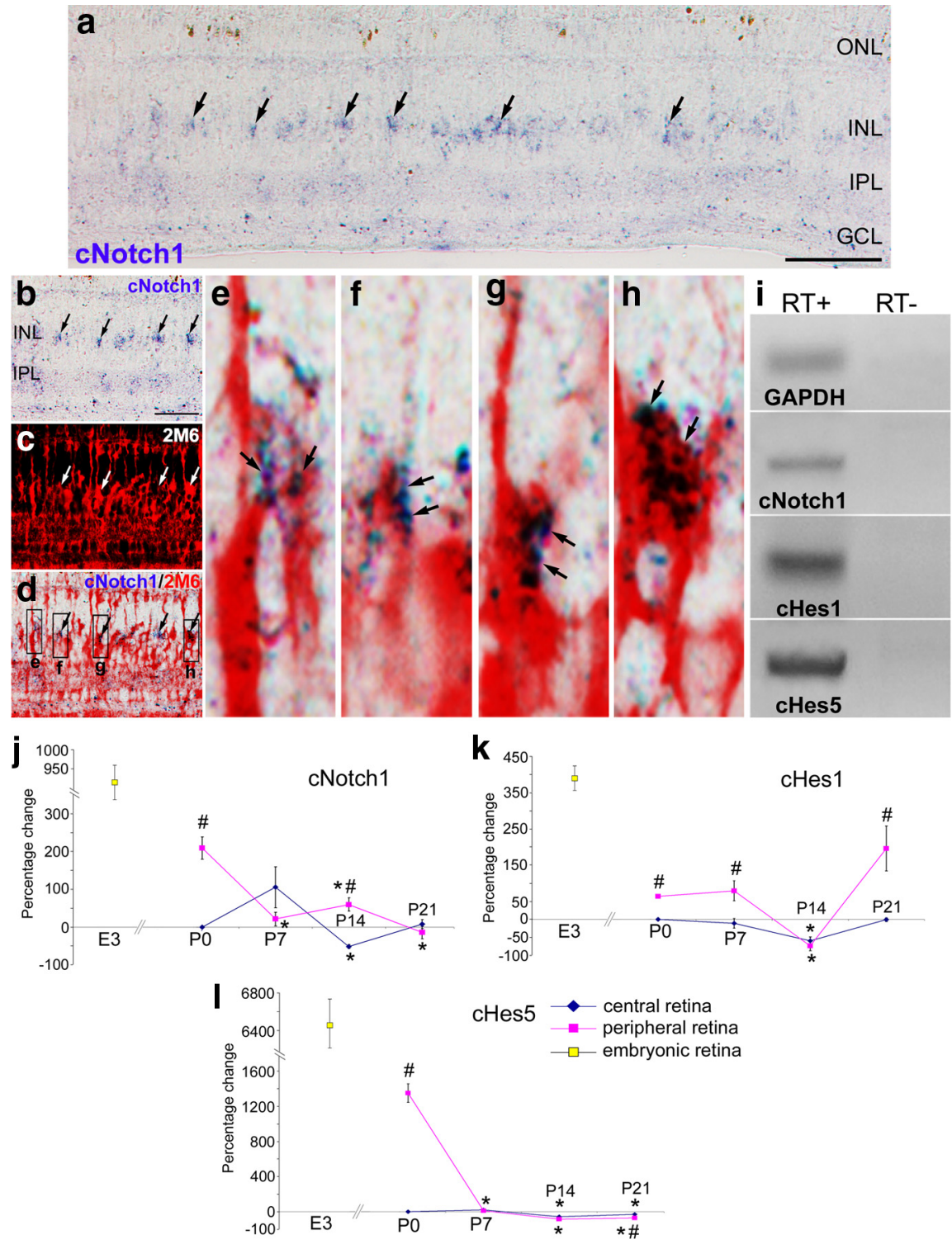

Figure 1. Components of the Notch-signaling pathway are expressed at low levels in the postnatal chicken retina. Vertical sections of the P7 retina were hybridized with riboprobes to CNotch1 $(\boldsymbol{a}, \boldsymbol{b} ;$ blue) and labeled for the glial marker 2M6 (c; red). d is a merged image of $\boldsymbol{b}$ and $\boldsymbol{c}$. $\boldsymbol{e}-\boldsymbol{h}$ are sevenfold enlargements of corresponding boxed areas in $\boldsymbol{d}$. Arrows in $\boldsymbol{b}-\boldsymbol{h}$ indicate cNotch1 and $2 \mathrm{M} 6$ double-positive cells. The scale bar $(50 \mu \mathrm{m})$ in $\boldsymbol{a}$ applies to $\boldsymbol{a}$ alone, and that in $\boldsymbol{b}$ applies to $\boldsymbol{b}-\boldsymbol{d}$. mRNA was harvested from retinas, reverse transcribed to generate CDNA, and amplified using $P C R(\boldsymbol{i})$ or real-time $P C R(\boldsymbol{j}-\boldsymbol{l})$. The $P C R$ products were run on an agarose gel, and stained with ethidium bromide ( $\boldsymbol{i}$ ). Reactions minus the reverse transcriptase enzyme (RT - ) were used as a negative control for each primer set. The percentage change in cDNA levels for cNotch1 $(\boldsymbol{j})$, cHes1 $(\boldsymbol{k})$, and cHes5 $(\boldsymbol{I})$ were calculated from $\mathrm{C}(\mathrm{t})$ values that were normalized to $\mathrm{GAPDH}$. Relative expression levels were measured for each mRNA at PO in central retina (set as baseline) and at P7, P14, and P21 in central and peripheral retina and E3 whole retina. Significance of difference $\left({ }^{*} p<0.005\right.$ between two time points within the same region, ${ }^{\#} p<0.01$ between central and peripheral retina at the same time point) was determined by using a two-factor ANOVA followed by a post hoc $t$ test. RT, Reverse transcriptase; INL, inner nuclear layer; IPL, inner plexiform layer.

Fixation, sectioning, and immunocytochemistry. Tissue dissection, fixation, cryosectioning, and immunolabeling were performed as described previously (Fischer et al., 2002b, 2009a). Working dilutions and sources of antibodies used in this study included the following: goat anti-Egr1 used at 1:1000 (AF2818; R\&D Systems); mouse anti-2M6 used at 1:100 (Dr. P. Linser, University of Florida, St. Augustine, FL); anti-Sox9 used at 1:2000 (AB5535; Millipore Bioscience Research Reagents); rabbit antiGFAP used at 1:2000 (Z0334; DakoCytomation); mouse anti-transitin used at 1:50 (EAP3; DSHB); mouse anti-vimentin used at 1:80 (H5; DSHB); mouse anti-Brn3a used at 1:50 (MAB1585; Millipore Bioscience Research Reagents); mouse anti-PCNA used at 1:1000 (M0879; DAKO); rat anti-BrdU used at 1:200 (OBT0030S; Serotec); mouse anti-BrdU used at 1:100 (G3B4; DSHB); rabbit anti-p38 MAPK used at 1:80 (12F8; Cell Signaling Technologies); rabbit anti-pCREB used at 1:600 (87G3; Cell Signaling Technologies); rabbit pERK1/2 used at 1:200 (137F5; Cell Signaling Technologies); and rabbit anti-Egr1 used at 1:400 (sc-110; Santa Cruz Biotechnology). Secondary antibodies included donkey anti-goat-Alexa488, goat anti-rabbit-Alexa488, goat anti-mouseAlexa488/568/647, goat anti-rat-Alexa488, rabbit anti-goat Alexa488, and goat antimouse-IgM-Alexa568 (Invitrogen) diluted to 1:1000 in PBS plus $0.2 \%$ Triton X-100. To permeabilize retinas for whole-mount labeling procedures, samples were frozen $\left(-80^{\circ} \mathrm{C}\right)$ and thawed $\left(20^{\circ} \mathrm{C}\right)$ three times before incubation with the antibody solution. Both primary and secondary antibodies were incubated overnight.

TUNEL. To identify dying cells that contained fragmented DNA, we used the TUNEL method. We used an In Situ Cell Death Kit (TMR red; Roche Applied Science), as per the manufacturer's instructions.

Photography, measurements, cell counts, and statistical analyses. Photomicrographs were taken by using a Leica DM5000B microscope equipped with epifluorescence and a 12 megapixel Leica DC500 digital camera. Confocal microscopy was done by using a Zeiss LSM 510 at the Hunt-Curtis Imaging Facility in The Ohio State College of Medicine. Images were optimized for color, brightness, and contrast, and double-labeled images overlaid by using Adobe Photoshop 6.0. Cell counts were made from at least six different animals, and means and SDs calculated on datasets. To avoid the possibility of region-specific differences within the retina, cell counts were consistently made from the same region of retina for each dataset.

For quantitative immunofluorescence all tissues were processed at the same time using single-batch preparations of all reagents. Immunofluorescence was quantified by using ImagePro 6.2. Identical illumination, microscope, and camera settings were used to obtain images for quantification. Areas $(800 \times 200$ pixels or $232 \times 54 \mu \mathrm{m}$ ) were sampled from 5.4 MP digital images. To avoid the possibility of regionspecific differences within the retina, areas were sampled from only central regions of retina. The areas were randomly sampled over the INL to selectively measure immunofluorescence intensity in the nuclei of the Müller glia, and to exclude immunofluorescence in neurons and glia in other retinal layers. Sampled areas were consistently obtained for sections of control and treated retinas from the same individual that were place consecutively (within $3 \mathrm{~mm}$ ) on glass slides to ensure equal exposure to reagents. Measurements were made for pixels with single-channel color values $(0=$ black, $255=$ saturated $)$ that were $>72$ for $\mathrm{pCREB}$ and $>65$ for p38 MAPK. Threshold levels were set to include immunofluorescence in Müller glia in both control and treated samples to allow cell-specific comparisons. The total area was calculated for regions with pixel intensities above threshold and the density sum was calculated as the total of pixel values for all regions that were above threshold. Measurements were made for retinal regions sampled from at least six different retinas for each experimental condition. These methods 
of quantitative immunofluorescence have proven to be accurate and highly reproducible (Fischer et al., 2009a,b, 2010; Ghai et al., 2009). Immunofluorescence was quantified by using ImagePro 6.2.

\section{Results}

Components of the Notch-signaling pathway are expressed at low levels in the postnatal chicken retina

To determine whether components of the Notch-signaling pathway are expressed in the postnatal chick retina, we used in situ hybridization, immunohistochemistry, and RT-PCR. We found that some cells in the inner nuclear layer (INL) of the P7 retina normally express cNotch 1 mRNA at low levels (Fig. 1a). These cells were immunoreactive for $2 \mathrm{M} 6$ (Fig. $1 b-h$ ), a monoclonal antibody that is known to selectively label Müller glia in the chicken retina (Linser et al., 1997; Fischer et al., 2009a). This pattern of expression is maintained in peripheral regions of the retina during the first 3 weeks of postnatal development. These findings suggest that Müller glia maintain low levels of Notch expression in undamaged, mature retina.

\section{Notch pathway gene expression varies} over time in the adult retina

Consistent with the findings of ISH studies, RT-PCR detected mRNA for cNotch1, cHes1, and cHes5 in undamaged postnatal chicken retina (Fig. 1i). The expression of Hes 1 and Hes5 is a readout of active Notch signaling (Kageyama and Ohtsuka, 1999; Nelson et al., 2006). These findings suggest that in addition to Notch expression, low levels of Notch signaling are sustained in mature Müller glia. PCR products were sequenced to verify the specificity of the reactions. We next assayed for changes in expression levels of Notch and related genes in central and peripheral regions of the postnatal retina and embryonic retina using real-time PCR. We found that the expression levels of cNotch1 are significantly higher in peripheral retina compared to levels in central retina at P0 and P14 (Fig. 1j). Further, expression levels of cNotch1 were reduced over time in the peripheral retina, compared to expression levels at P0. Similar to cNotch1, there was a tendency for $\mathrm{cHes} 1$ and $\mathrm{cHes} 5$ expression to decrease from P0 to P14. However, expression levels of cHes 1 in central and peripheral retina increased again at P21. cHes1 expression was significantly higher in peripheral retina compared to central retina at $\mathrm{P} 0, \mathrm{P} 7$, and $\mathrm{P} 21$. cHes1 expression in central retina remained relatively low and unchanged throughout the first 3 weeks of postnatal development (Fig. $1 k$ ). By comparison, cHes5 expression declined rapidly in peripheral retina from $\mathrm{P} 0$ through P7, whereas cHes5 remained low, and unchanged, in central retina throughout postnatal development similar to cHes1 expression (Fig. 1l). As expected, expression levels of cNotch1, cHes1, and cHes5 were much higher in embryonic retina compared to postnatal retina (Fig. $1 j-l$ ). Interestingly, cHes5 expression in embryonic retinas was 64-fold higher than that in central retina at P0 (Fig. 1l). The differences in expression levels of cNotch1 and cHes1 in embryonic and postnatal retinas were modest compared to differences seen for levels of cHes5 (Fig. $1 j, k)$. These findings suggest that even though $\mathrm{cHes} 1$ and $\mathrm{cHes} 5$ are both downstream effectors of Notch, their expression may be differentially regulated across time and in different regions of the retina.

\section{Inhibition of Notch signaling in the retina does not affect glial reactivity}

To determine whether Notch signaling influences Müller glia in mature retina, we inhibited Notch signaling by using a $\gamma$-secretase inhibitor, DAPT. Using conventional PCR (Fig. $2 a$ ) and real-time PCR (Fig. 2b), we observed that DAPT significantly reduced the expression levels of cNotch1, cDelta1, cDll4, cHes1, and cHes2 in the retina, whereas levels of cHes5 were unaffected (Fig. 2b). However, there was no apparent change in the expression levels of Sox9 (Fig. $2 c, d$ ), GFAP (Fig. 2e,f), transitin (Fig. 2g,h), and vimentin (Fig. 2i,j), as determined by real-time PCR (data not shown) and immunohis- 

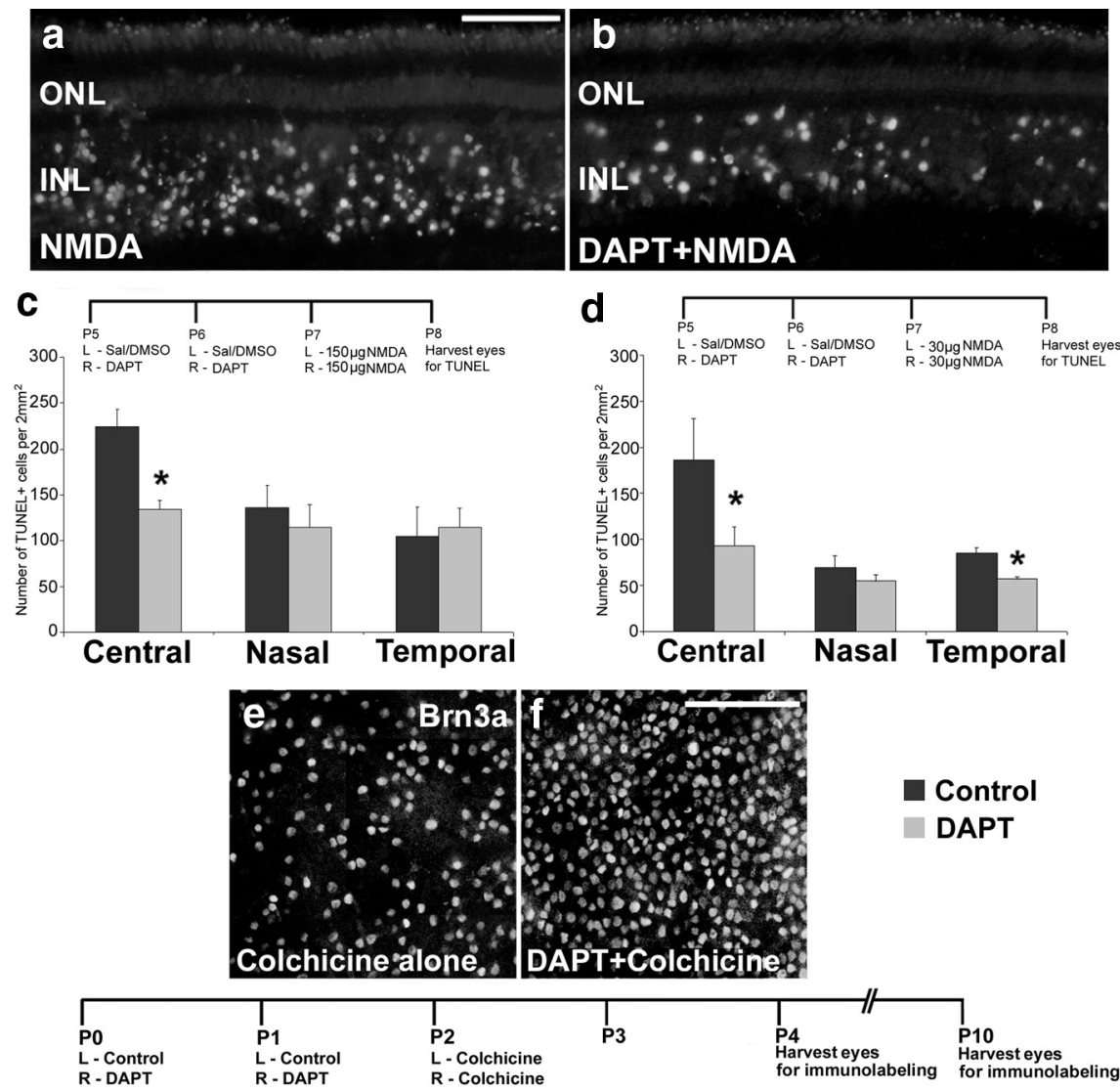

g

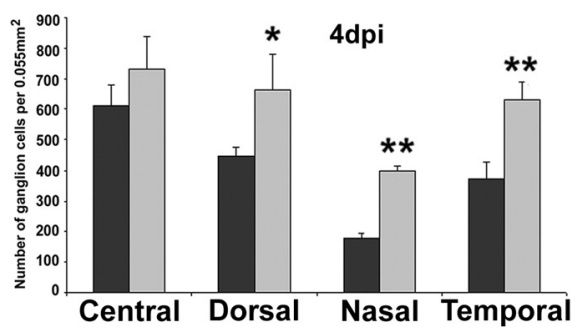

h

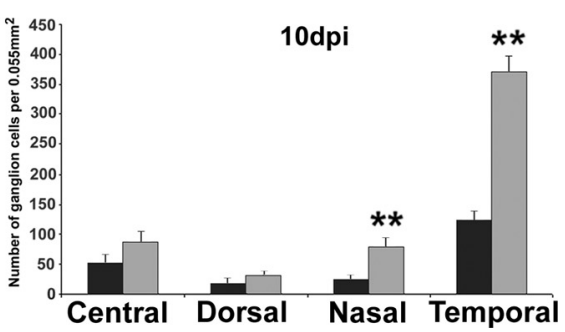

Figure 3. Inhibition of Notch signaling before retinal injury decreases cell death and increases neuronal survival. $\boldsymbol{a}, \boldsymbol{b}$, Representative images of TUNEL-positive nuclei in vertical sections of retinas for eyes that were treated with vehicle before NMDA (a) or DAPT before NMDA (b). $\boldsymbol{c}, \boldsymbol{d}$, Histograms illustrate the mean ( \pm SEM) number of dying cells in the INL in central, nasal, or temporal regions of the retina $(\boldsymbol{c}, \boldsymbol{d})$. Numbers of TUNEL + cells were counted in retinas treated with $150 \mu \mathrm{g}$ of NMDA (c) or $30 \mu \mathrm{g}$ of NMDA (d) with and without DAPT pretreatment. $\boldsymbol{e}, \boldsymbol{f}$, Representative images of whole-mounted retinas that were treated with vehicle before colchicine $(\boldsymbol{e})$ or DAPT before colchicine $(\boldsymbol{f})$. Retinas were labeled with antibodies to Brn3a to identify ganglion cells. Histograms illustrate the mean ( \pm SEM) number of ganglion cells per $0.55 \mathrm{~mm}^{2}$ in central, dorsal, nasal, or temporal regions of the retina $(\boldsymbol{g}, \boldsymbol{h})$. Datasets were obtained from 6 animals per treatment, per time point. Significance of difference $\left({ }^{*} p<0.05,{ }^{* *} p<0.01\right)$ was determined by using a two-tailed, unpaired Student'st test. ONL, Outer nuclear layer; INL, inner nuclear layer. The scale bar $(50 \mu \mathrm{m})$ in $\boldsymbol{a}$ applies to $\boldsymbol{a}$ and $\boldsymbol{b}$, and that in $\boldsymbol{f}$ applies to $\boldsymbol{e}$ and $\boldsymbol{f}$.

tochemistry. These genes are normally expressed by Müller glia in the mature retina. Transitin and GFAP are known to be upregulated in reactive Müller glia (Fischer and Omar, 2005). These findings suggest that DAPT does not induce reactivity in Müller glia.

\section{Intraocular injections of a Notch inhibitor before retinal} injury reduces cell death

Given that some of the Müller glia normally express cNotch1 at low levels and Notch signaling is upregulated in damaged retinas (Hayes et al., 2007), we sought to determine whether blocking Notch signaling influenced glial responses to acute damage. NMDA-induced retinal damage is known to stimulate the proliferation of Müller glia in birds and rodents (Fischer and Reh,

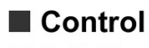

DAPT

2001; Karl et al., 2008); this proliferation is thought to be an integral step in the transdifferentiation of Müller glia (Fischer and Reh, 2003; Lamba et al., 2009). We found that inhibition of Notch signaling in Müller glia before NMDA treatment caused a decrease in the number of proliferating glia (data not shown).

To test whether inhibition of Notch signaling influenced the ability of Müller glia to protect retinal neurons from damage, we injected DAPT before NMDA or colchicine treatment and harvested the retinas 1, 2, or $10 \mathrm{~d}$ later. NMDA results in excitotoxic death of amacrine and bipolar cells (Fischer et al., 1998), and colchicine results in death of ganglion cells (Morgan, 1981; Morgan and Spooner, 1983; Fischer et al., 1999). We found that two consecutive daily doses of $865 \mathrm{ng}$ of DAPT significantly reduced NMDA-induced cell death by $43 \%$ in central regions of the retina with no change in cell death in nasal or temporal retina (Fig. $3 a-c$ ). With a lower dose of NMDA (30 $\mu \mathrm{g})$, DAPT pretreatment lowered the abundance of TUNEL+ cells, with a $50 \%$ decrease in cell death in central regions, a $43 \%$ decrease in cell death in temporal regions, and no significant changes in cell death in nasal regions of the retina (Fig. $3 d$ ).

We next assayed for the survival of ganglion cells in colchicine-damaged retinas by probing for Brn3a, a homeodomain transcription factor that is expressed by $\sim 98 \%$ of the ganglion cells (Liu et al., 2000; Badea et al., 2009). DAPT before colchicine treatment resulted in 55\%, $100 \%$, and $62 \%$ more surviving ganglion cells in dorsal, nasal, and temporal regions of the retina, respectively, at $4 \mathrm{~d}$ after injury (Fig. $3 e-g$ ). However, in colchicine-treated retinas, numbers of dying ganglion cells are maximal between 3 and $4 \mathrm{~d}$ after treatment (Fischer and Reh, 2002; Fischer et al., 2008). Thus, we next assayed for ganglion cell survival at $10 \mathrm{~d}$ after treatment when the damaging effects of colchicine had subsided. With DAPT pretreatment, elevated survival of ganglion cells was seen at $10 \mathrm{~d}$ after injury in the nasal retina (Fig. $3 h$ ). However, the most prevalent survival-promoting effects for DAPT were observed in temporal regions of the retina with a near threefold increase in surviving ganglion cells compared to retinas $10 \mathrm{~d}$ after treatment with colchicine alone (Fig. 3 h). Collectively, our results suggest that DAPT enhances neuronal survival when administered before injury by inhibiting Notch signaling in Müller glia.

Notch is upregulated in retinas treated with growth factors insulin and FGF2

Previous studies have shown that Notch is upregulated in Müller glia after retinal injury (Hayes et al., 2007; Yurco and Cameron, 
2007). However, it remains uncertain whether glial increases in Notch signaling in response to damage function exclusively in the process of proliferation and transdifferentiation or in the process of damage-induced reactive gliosis. Accordingly, we tested whether Notch is upregulated in Müller glia when stimulated to proliferate and transdifferentiate in the absence of injury. We have previously shown that three consecutive daily doses of insulin and FGF2 result in a peripheralto-central wave of proliferation within the retina and that these dividing cells are Müller glia (Fischer et al., 2002b). In response to insulin and FGF2, cNotch1 was upregulated in proliferating Müller glia (Fig. $4 a-g$ ). This effect was also seen in retinas treated with four consecutive daily doses of IGF1 and FGF2 (data not shown). Saline injections did not influence cNotch1 expression or the proliferation of cells (Fig. $4 h-j$ ).

Using real-time PCR, we observed that three consecutive daily doses of insulin and FGF2, a dose regimen known to stimulate the proliferation and transdifferentiation of Müller glia (Fischer et al., 2002b), induced an upregulation of cNotch1 and related genes. We found a 3.5-fold increase in cNotch1, 15-fold increase in cDelta1, 12-fold increase in cDll4, 4.5-fold increase in cHes5, and a 1.5-fold increase in cHes1 (Fig. $5 a$ ). We also found significant increases in the expression of other Notch-related genes such as a twofold increase in the ligand cJagged, 4-fold increase in cHey1, 12-fold increase in cHey2, but no change in the proneural gene asclla (Fig. $5 a$ ). We next measured levels of cNotch 1 and related genes in retinas treated with only 2 consecutive daily injections of insulin and FGF2, a dose regimen that does not stimulate the proliferation and transdifferentiation of Müller glia (Fischer et al., 2002b, 2004b). Surprisingly, we found that 2 doses of insulin and FGF2 decreased levels of Notch1 and related genes (Fig. 5b). Decreased levels of expression were observed for cNotch1 (0.6-fold), cDeltal (1.2-fold), cDll4 (1.1fold), cJagged (1.0-fold), cHes1 (0.8-fold), cHes5 (0.4-fold), cHey1 (0.8-fold), cHey2 (0.5-fold), and asclla (0.8-fold) (Fig. $5 b)$. To determine whether expression levels of Notch and related genes were influenced by FGF or insulin alone, we injected either growth factor separately and harvested retinas $24 \mathrm{~h}$ after the last injection. Two consecutive daily doses of FGF2 induced a 6-fold increase in cNotch 1 expression, 4.5-fold increase in cDll4, 5.7fold increase in cJagged, 3-fold increase in cHes5 and cHeyl, 5 -fold increase in cHey2, and small decreases in cDelta1, cHes1, and asclla expression (Fig. 5c). Two consecutive daily doses of insulin, however, did not affect levels of cNotch1, and resulted in modest but significant decreases in expression levels of cNotch1 (0.2-fold), cDelta1 (0.6-fold), cHes1 (1.1-fold), cHes5 (0.5-fold), cHey2 (0.6-fold), and asclla (0.3-fold) (Fig. $5 d$ ). Interestingly, insulin induced small but significant increases in expression levels of cDll4 (0.4-fold), cJagged (0.15-fold), and cHey1 (0.2-fold)
(Fig. 5d). Collectively, these findings suggest that sustained exposure to the combination of insulin and FGF2 enhances expression of Notch and related genes, whereas short-term exposure to the combination of insulin and FGF2 decreases expression of Notch and related genes. By comparison, insulin alone tended to decrease Notch and related genes, whereas FGF2 alone tended to increase levels of Notch1 and related genes.

\section{FGF2 upregulates effectors of MAPK signaling in Müller glia} and stimulates proliferation in damaged retinas

We have previously shown that the neuronal damage caused by $300 \mu \mathrm{g}$ of NMDA induces the accumulation of pCREB in Müller glia at 2-3 d after injury, and that inhibition of FGF receptors and MEK suppresses the proliferation of Müller glia (Fischer et al., 2009a). In the absence of damage, FGF2 induces the accumulation of MAPK effectors, including pERK1/2, cFos, Egr1, pCREB, and p38 MAPK in Müller glia, whereas retinal neurons appear unaffected (Fischer et al., 2009b). It is also known that $150 \mu \mathrm{g}$ of NMDA causes some cell death, but fails to induce Müller glial proliferation as is elicited by the maximum dose $(300 \mu \mathrm{g})$ (Fischer and Reh, 2001; Fischer et al., 2004a) (Fig. 6i). Hence, we tested whether FGF2 stimulated the accumulation of MAPK effectors and proliferation of Müller glia after moderate levels of retinal damage. We administered $150 \mu \mathrm{g}$ of NMDA into eyes followed by two daily doses of saline (control) or FGF2 (treat- 


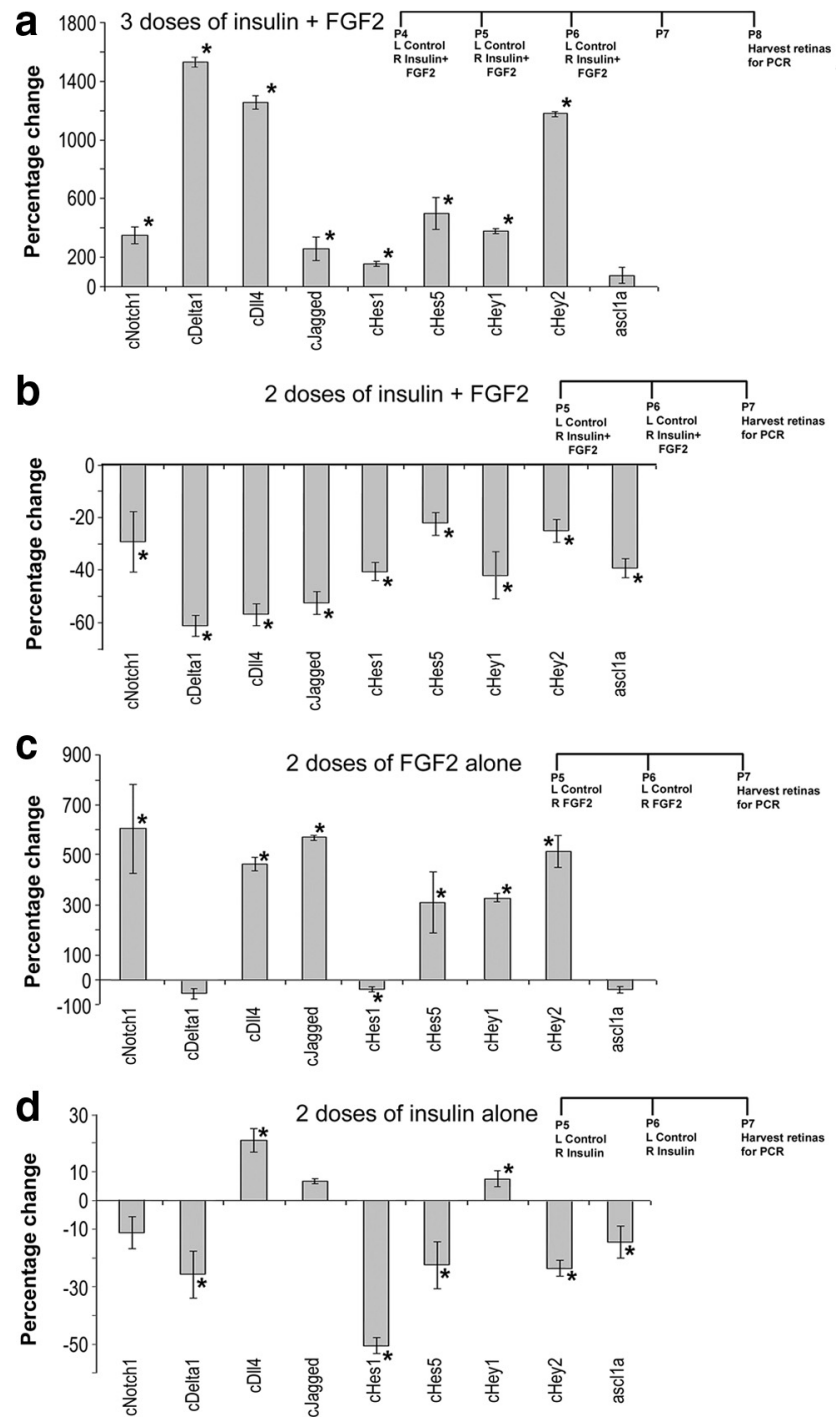

Figure 5. Notch and related genes are upregulated in retinas treated with insulin and FGF2. $\boldsymbol{a}-\boldsymbol{d}$, mRNA was harvested from retinas obtained from eyes treated with saline (control) or 3 consecutive daily doses of insulin and FGF2 (a), saline (control) or 2 consecutive daily doses of insulin and FGF2 (b), saline (control) or FGF2 alone (c), and saline (control) or insulin alone (d). Individual doses of insulin and FGF2 were 1000 and $250 \mathrm{ng}$, respectively. mRNA was reverse transcribed to generate CDNA and amplified using real-time $P C R$. Ct values were normalized to GAPDH, and the fold difference between control and treated samples was determined using the $\Delta \mathrm{Ct}$ method and represented as a percentage change $(\boldsymbol{a}-\boldsymbol{d})$. Significance of difference $\left(^{*} p<\right.$ 0.03) was determined by using a two-tailed, unpaired Student's $t$ test. RT, Reverse transcriptase.

ment) and BrdU and assayed for proliferating cells. We found that FGF2 administered after NMDA greatly enhanced Müller glial proliferation (Fig. 6a-i). Sox9-positive Müller glia expressed high levels of PCNA and accumulated BrdU in retinas treated with FGF2 following NMDA (Fig. $6 e-h$ ). Although low levels of PCNA were detected in Müller glia in retinas treated with NMDA alone, few of these cells accumulated BrdU and levels of PCNA appeared much lower than those seen in Müller glia in retinas treated with NMDA and FGF2 (Fig. $6 a-h$ ). Thus, the Müller glia with low levels of PCNA likely were not proliferating, and only those cells labeled for BrdU and high levels of PCNA were counted as proliferating. We also found that FGF2 increased levels of p38 MAPK and pCREB in Müller glia (Fig. 6j-u), but not pERK, Egr1, and cFos (data not shown). This accumula- tion of pCREB and p38 MAPK in Müller glia suggests that MAPK signaling has been activated by FGF2. Thus, FGF2 selectively stimulates MAPK signaling in Müller glia and induces proliferation of these glia after moderate levels of retinal injury.

\section{Inhibition of Notch reduces FGF2-induced accumulations of p38 MAPK and pCREB in Müller glia}

Some studies have indicated that interactions exist between the Notch and the FGF2-MAPK pathway in the developing nervous system (Faux et al., 2001; Ota and Ito, 2006; Wahl et al., 2007; Saravanamuthu et al., 2009). However, such an interaction has not been demonstrated in the retina. Since components of the Notch-signaling pathway were upregulated by FGF2 (Figs. 4, 5), we tested whether Notch reciprocally influences FGF2-MAPK signaling. Specifically, we tested whether Notch activity is required for the upregulation of pCREB and p38 MAPK in damaged retinas treated with FGF2. After treatment with $150 \mu \mathrm{g}$ of NMDA, two consecutive daily doses of FGF (control) or FGF and DAPT (treatment) were injected into eyes that were harvested $24 \mathrm{~h}$ after the last dose. The FGF-induced accumulations of pCREB (Fig. 7a,c) and p38 MAPK (Fig. 7g) in the Müller glia in damaged retina were significantly reduced by DAPT (Fig. $7 b, d-f, h-j)$. We did not observe any change in expression levels of FGFR1 or FGFR3 mRNA in this paradigm (data not shown), suggesting that DAPT treatment acts on downstream components of the FGF-MAPK pathway rather than inhibiting receptor-expression levels. It should be noted that we were unable to detect significant levels of FGFR2 in the retina consistent with previous reports (Fischer et al., 2009a), and that FGFR4 may not exist in chicken retina (M. A. Scott and A. J. Fischer, unpublished observation).

Our results suggest that there are interactions between the Notch pathway, p38 MAPK, and pCREB, but not ERK1/2, Egr1, or cFos. However, pERK and associated immediate early genes cFos and Egr 1 accumulate soon $(<2 \mathrm{~d})$ after acute damage in the retina, whereas p38 MAPK and pCREB accumulate later $(>2 \mathrm{~d})$ (Fischer et al., 2009a). Hence, changes in ERK1/2, cFos, and Egr1 expression may have been missed because our studies focused on the effects of DAPT at $3 \mathrm{~d}$ after damage.

\section{Inhibition of Notch activity reduces FGF-induced proliferation of Müller glia}

Since DAPT inhibited the accumulation of p38 MAPK and pCREB in Müller glia (Fig. 7), we tested whether DAPT influenced FGF2-induced glial proliferation in damaged retinas. To do this, we injected $150 \mu \mathrm{g}$ of NMDA into eyes at P4 followed by 2 injections of FGF2 (control) or FGF2 and DAPT (treatment) at P5 and P6, and BrdU at P6 to label proliferating cells. Eyes were harvested at P7, $3 \mathrm{~d}$ after the excitotoxic insult. As expected, FGF2 stimulated Müller glial proliferation after NMDA injury to levels similar to those seen $3 \mathrm{~d}$ after $300 \mu \mathrm{g}$ of NMDA alone (compare Figs. 6i, 8i). Interestingly, DAPT blocked the FGF2-induced proliferation of Müller glia (Fig. $8 e-h$ ), suggesting that FGF2/MAPK signaling requires active Notch signaling to stimulate the proliferation of Müller glia in damaged retinas.

In another paradigm, we injected FGF2 (control) or FGF2 and DAPT (treatment) at P4 and P5 followed by $150 \mu \mathrm{g}$ of NMDA into eyes at P6 and BrdU at P7 to label proliferating cells. Eyes were harvested $4 \mathrm{~h}$ after the BrdU injection. Similar to the results seen when FGF2 and DAPT were injected after NMDA, we observed that DAPT blocked the proliferation-inducing effects of FGF2 when applied before NMDA (Fig. 8i). 
To determine whether inhibition of Notch signaling in undamaged retinas influences the ability of Müller glia to reenter the cell cycle, we injected three consecutive doses of DAPT at P4, P5, and P6, insulin and FGF2 at P7, P8, and P9, and BrdU at P9 and P10. Eyes were harvested at P11. Injections of DAPT before insulin and FGF2 reduced the proliferation of Müller glia in central regions of the retina by $50 \%$, but did not reduce proliferation in nasal or temporal regions of the retina (supplemental Fig. 1, available at www. jneurosci.org as supplemental material). This finding suggests that inhibition of Notch signaling in normal, undamaged retinas reduces the potential of Müller glia to become proliferating progenitor-like cells. Collectively, these findings indicate that Notch signaling is required for FGF2 to stimulate glial proliferation.

\section{Discussion}

We find that components of the Notchsignaling pathway are expressed by a subset of Müller glia in undamaged retina where this pathway regulates neuroprotective and proliferative properties of the glia. The Notch pathway has been extensively studied in the developing retina, but few studies have addressed its function in the mature retina. During embryonic development, Notch and its effectors are highly expressed by retinal progenitors in the chick retina at E4-E4.5 (Austin et al., 1995; Nelson et al., 2006) and E5.5 (Kubo and Nakagawa, 2009) and in the rat retina from E12.5 to P0 (Bao and Cepko, 1997). Over time, Notch expression is confined to the peripheral retina (Bao and Cepko, 1997) and is thought to be completely absent in central retina, although some expression in the adult retina has been reported (Ahmad et al., 1995; Hayes et al., 2007). Our findings indicate that low levels of Notch expression are maintained in chick retina, long after the completion of histogenesis (Prada et al., 1991; Ghai et al., 2008). Further, we find elevated levels Notch signaling in peripheral retina compared to levels seen in central regions of retina. Our findings indicate that most, if not all, of the Notch expression is restricted to Müller glia. Moreover, Müller glia appear to be heterogeneous with respect to Notch expression. The Müller glia that maintain Notch signaling may be the cells with the greatest potential for transdifferentiation; it is expected that sustained Notch signaling may preserve progenitor phenotype.

Consistent with our findings, single-cell gene expression analysis of Müller glia in rodents revealed highly enriched expression of genes associated with Notch signaling, such as Nrarp, Notch1, Notch2, Cntn1, and Hes1(Roesch et al., 2008). The cell types that express Notch ligands in the mature retina remain unknown, but may include neurons. In the developing retina, Deltal is expressed primarily in progenitor cells, Dll4 is expressed in newly
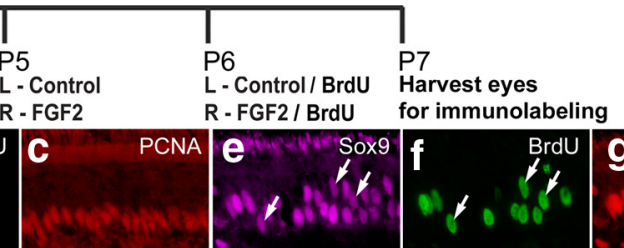

e $\quad$ Sox 1 if
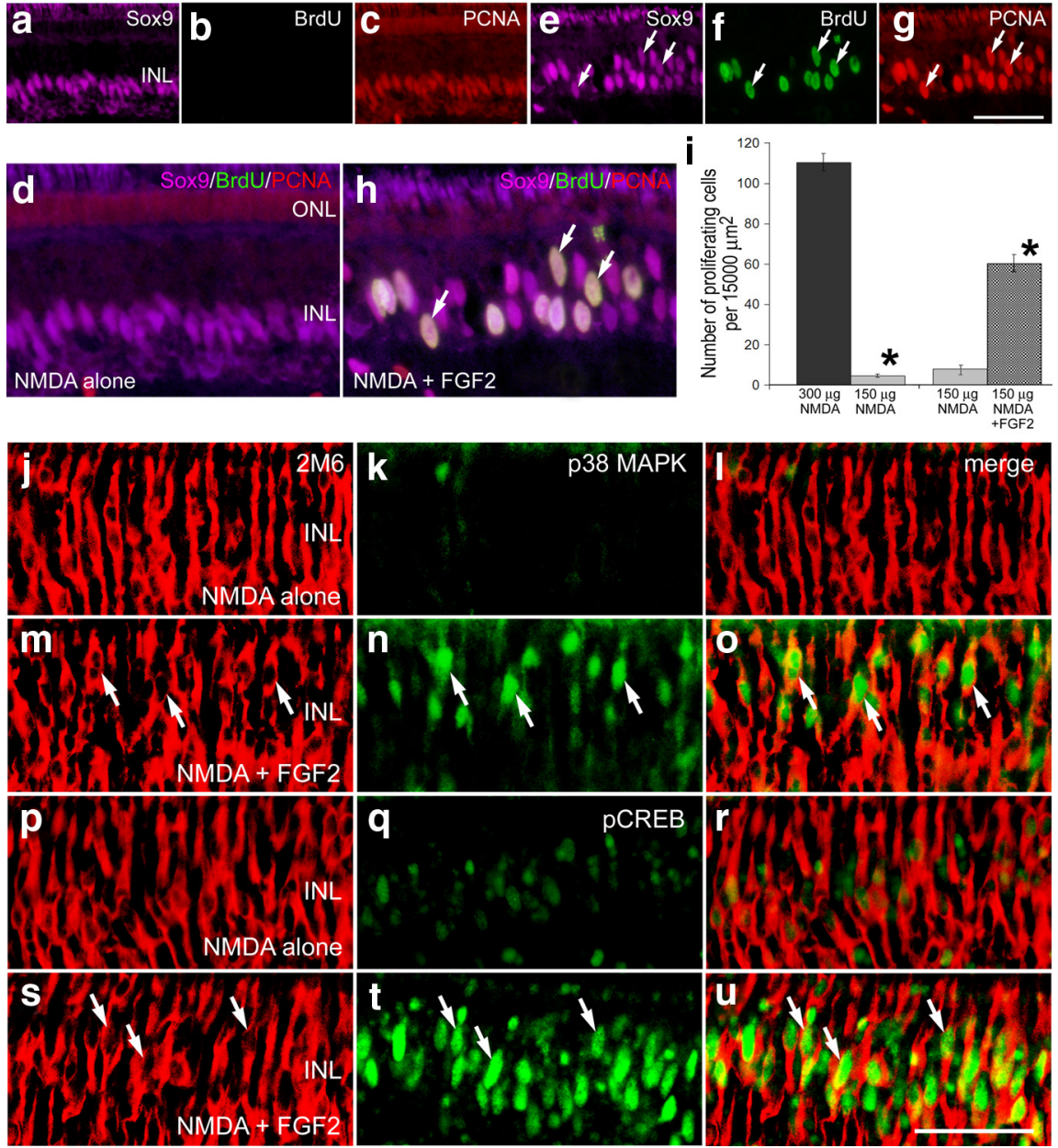

Figure 6. FGF2 induces the proliferation of Müller glia in injured retinas. Eyes were treated with $150 \mu \mathrm{g}$ of NMDA at P4 followed by two consecutive daily doses of saline (control) or $250 \mathrm{ng}$ of FGF2 (treated) at P5 and P6 and a dose of BrdU at P6, and harvested $24 \mathrm{~h}$ later at P7. $\boldsymbol{a}-\boldsymbol{c}, \boldsymbol{e}-\boldsymbol{g}$, Vertical sections of the P7 retina were labeled for Sox9 ( $\boldsymbol{a}, \boldsymbol{e}$; magenta), BrdU (b, $\boldsymbol{f}$; green), and PCNA (c, $\boldsymbol{g}$; red). $\boldsymbol{d}$ is a merged image of $\boldsymbol{a}-\boldsymbol{c} . \boldsymbol{h}$ is a merged image of $\boldsymbol{e}-\boldsymbol{g}$. $\boldsymbol{d}$ and $\boldsymbol{h}$ are 3.5 -fold enlargements of $\boldsymbol{a}-\boldsymbol{c}$ and $\boldsymbol{e}-\boldsymbol{g}$, respectively. BrdU- and PCNA-positive cells per $15,000 \mu \mathrm{m}^{2}$ of retinas. $\boldsymbol{j}-\mathbf{0}, \boldsymbol{p}-\boldsymbol{u}$, Vertical sections from control and treated retinas were abeled for 2 M6 (red) and p38 MAPK (green;j- 0 ) or pCREB (green; $\boldsymbol{p}-\boldsymbol{u}$ ). Significance of difference $\left({ }^{* *} p<0.001\right.$ ) was determined by using a two-tailed, unpaired Student's $t$ test. Arrows indicate 2M6-positive Müller glia labeled for p38 MAPK ( $\boldsymbol{m}-\boldsymbol{0}$ ) or pCREB $(\boldsymbol{s}-\boldsymbol{u})$. INL, Inner nuclear layer. The scale bar $(50 \mu \mathrm{m})$ in $\boldsymbol{g}$ applies to $\boldsymbol{a}-\mathbf{c}$ and $\boldsymbol{e}-\boldsymbol{g}$, and that in $\boldsymbol{u}$ applies to $\boldsymbol{j}-\boldsymbol{u}$.

differentiated neurons (Nelson and Reh, 2008), and Jagged is expressed in postmitotic ganglion cells (Valsecchi et al., 1997). It is possible that microglia may express Notch ligands; these cells have been shown to interact with Müller glia during retinal injury (Harada et al., 2002). Another possibility is that Notch ligands are expressed by Müller glia, although convincing data to support this hypothesis is currently lacking. Consistent with this hypothesis, FGF2 increases retinal levels of Delta1, Dll4, and Jagged (current study), and FGF2 acts directly on Müller glia (Fischer et al., 2009b). Further studies are required to unambiguously identify the cell types that express Notch ligands in the mature retina.

We report here that Notch signaling in mature Müller glia may dampen the neuroprotective properties of these glia; inhibition of Notch signaling increases neuronal survival. Müller glia protect retinal neurons against excitotoxic cell death via the up- 

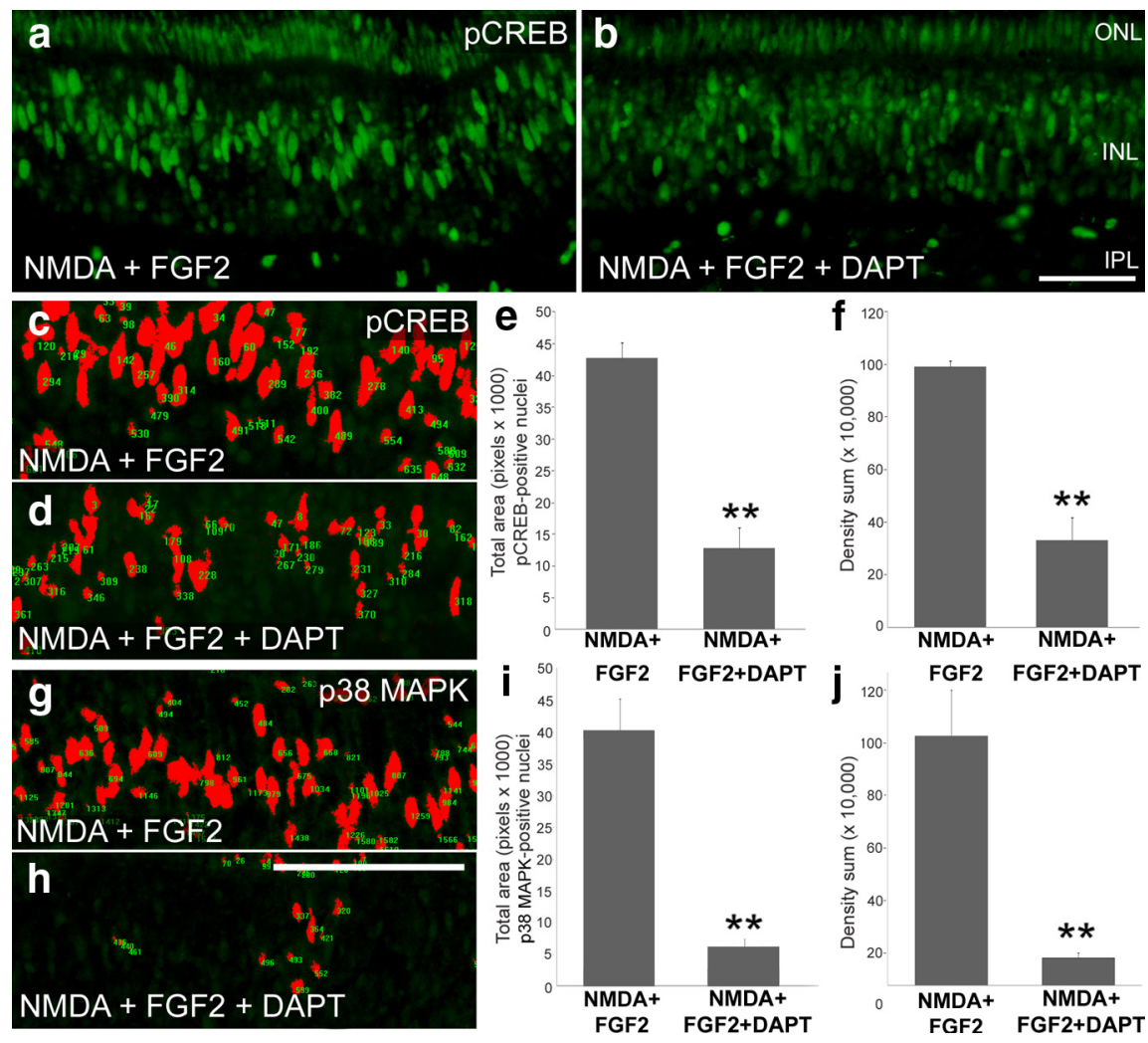

Figure 7. Inhibition of Notch signaling reduces levels of pCREB and 38 MAPK in Müller glia. DAPT suppresses the accumulation of 38 MAPK and pCREB in Müller glia that results from NMDA + FGF2 treatment. Images were obtained using identical camera exposures and microscope illumination settings. Retinas were processed for immunolabeling after treatment with $150 \mu \mathrm{g}$ of NMDA and two consecutive daily doses of $250 \mathrm{ng}$ of FGF2 (control) or $250 \mathrm{ng}$ of FGF2 $+865 \mathrm{ng}$ of DAPT (treated). As described in the Materials and Methods, ImagePro 6.2 was used to obtain measurements of total area for pixel intensities $>72$ for pCREB and $>65$ for p 38 MAPK ( $0=$ black, $255=$ saturated green), and the density sum. The small numbers and red areas in $\boldsymbol{c}, \boldsymbol{d}, \boldsymbol{g}$, and $\boldsymbol{h}$ indicate the pixels designed by ImagePro 6.2 that met the threshold criteria. Means and SDs are displayed in histograms for total area $(\boldsymbol{e}, \boldsymbol{i})$ and density sum $(\boldsymbol{f}, \boldsymbol{j})$ for areas with pixel values above threshold. Significance of difference $\left(^{* *} p<0.001\right)$ was determined by using a two-tailed, unpaired Student's $t$ test. ONL, Outer nuclear layer; INL, inner nuclear layer; IPL, inner plexiform layer. The scale bar $(50 \mu \mathrm{m})$ in $\boldsymbol{b}$ applies to $\boldsymbol{a}$ and $\boldsymbol{b}$, and that in $\boldsymbol{h}$ applies to $\boldsymbol{c}-\boldsymbol{h}$.

take and degradation of glutamate, release of neurotrophic factors, and the secretion of the antioxidant glutathione (Bringmann et al., 2006). However, the Notch-dependent mechanisms that regulate the ability of Müller glia to support neuronal survival remain uncertain. Further, ganglion cell death stimulates gliosis in Müller cells (Bringmann and Reichenbach, 2001; Inman and Horner, 2007). However, there currently is no evidence that Notch signaling influences the gliotic phenotypes of the Müller cells. The mechanisms of communication between ganglion cells and Müller glia remain uncertain, but could involve Notch signaling and perhaps Shh signaling (Fischer and Scott, unpublished observations).

Our results indicate that Notch signaling in Müller glia supports the survival of ganglion cells. In addition, our findings indicate that there are regional differences in cell death and survival when Notch signaling is inhibited by DAPT. In the colchicineinjury model, neuroprotection is enhanced by DAPT in peripheral retinal regions compared to that seen in central regions of the retina. This could result from increased levels of Notch signaling in Müller glial in peripheral retinal regions. This notion is consistent with reports that Müller glia in peripheral retina mature more slowly than those in central retina (Harman and Beazley, 1989; Anezary et al., 2001; Ghai et al., 2008). Further, Müller glia in peripheral regions of the retina transdifferentiate in greater numbers than the glia found in central regions of the retina (Fischer and Reh, 2003; Fischer et al., 2004b). Thus, the survivalpromoting effects of DAPT in peripheral regions of the retina may reflect elevated levels of Notch signaling in Müller glia in peripheral retina compared to levels maintained in glia in central retina.

The link between Notch signaling and FGF-MAPK signaling has not been established previously in the retina. We have shown that FGF2 stimulates neuroprotection and proliferation of Müller glia in damaged retinas (Fischer et al., 2009b). Here we find that glial proliferation mediated through FGF2/MAPK signaling requires Notch signaling. However, FGF2mediated neuroprotection by Müller glia is not influenced by blocking Notch signaling (supplemental Fig. 2, available at www.jneurosci.org as supplemental material). This indicates that the Notchdependent mechanism by which FGF2 stimulates glial proliferation is distinct from the mechanism by which FGF2 enhances neuroprotection through Müller glia. Further, the interaction of MAPK and Notch signaling in stimulating glial proliferation may involve a feedforward loop. For example, the expression of Notch and related genes is increased in Müller glia, in the absence of damage, by insulin and FGF2. Consistent with our findings, interactions between FGF2/ MAPK and Notch are known to exist in the developing nervous system. FGF2 upregulates Notch expression in neuroepithelial precursor cells and acts synergistically with Notch to inhibit differentiation in these cells (Faux et al., 2001). Further, FGF2 promotes gliogenesis and suppresses Ngn2 in trunk neural crest cells by increasing expression of Notch 1 and Delta1 (Ota and Ito, 2006). During somitogenesis, FGF/MAPK signaling controls the oscillating expression levels of Notch pathway genes in presomitic mesoderm (Wahl et al., 2007). In the eye, FGF2induced expression of Jag1 and Notch2 was decreased by DAPT, demonstrating the dependence of FGF2 signaling on Notch for normal lens development (Saravanamuthu et al., 2009). Collectively, these reports support our findings that FGF2/MAPK signaling requires Notch to stimulate the dedifferentiation and proliferation of Müller glia.

From previous studies, we know that the Müller glial proliferation is potently stimulated by FGF2 only when combined with insulin (Fischer et al., 2002b), IGF1 (current studies), or low levels of NMDA-induced damage (Fischer et al., 2009b). Thus, FGF and MAPK signaling facilitate the proliferation of Müller glia, but are not sufficient to drive this process unless coupled with insulin/IGF1 or neuronal damage. Further, we find that insulin or FGF2 alone have different effects upon Notch pathway genes. The combination of insulin and FGF2 act synergistically to dramatically increase levels of genes associated with the Notch pathway; an effect not seen with either factor alone. Expression of Notch ligands and effectors also 
varied greatly between treatments. These differences could be explained by different sites of action. FGFR1 may be expressed by Müller glia, whereas the insulin receptor is not detectable in the retina (Fischer et al., 2009b). Additionally, IGF1R is expressed by microglia and novel nonastrocytic inner retinal glia-like (NIRG) cells (Fischer et al., 2010). Thus, FGF-induced Notch signaling may be a direct effect on Müller glia, whereas insulin/IGF1 may suppress Notch signaling in Müller glia through intermediate cells and signals. Together, insulin and FGF2 have combined effects on Notch signaling through direct effects on Müller glia and indirect effects through microglia and/or NIRG cells. We propose that the synergistic influence of FGF2 and insulin/IGF1 on expression of Notch and related genes in Müller glia is needed to stimulate proliferation without neural damage.

Collectively, our findings indicate that MAPK signaling alone is not sufficient to induce glial proliferation; Notch signaling and/or damage-derived signals are required. Interestingly, Notch activity is required for the proliferation-enhancing effects of FGF/MAPK signaling, but decreases neuroprotection in the retina, unlike FGF/MAPK signaling. Our data suggest that neuroprotection through inhibition of Notch signaling does not involve suppression of MAPK signaling in Müller glia since MAPK effectors such as p38, MAPK, pERK1/2, and pCREB are undetectable in mature Müller glia in undamaged retinas (Fischer et al., 2009a,b). Inhibition of Notch activity increased the neuroprotective capacity of Müller glia, similar to activation of FGF2-MAPK signaling (Fischer et al., 2009b). Further, Notch signaling in the retina promotes progenitor-like characteristics in Müller glia, similar to FGF/MAPK signaling. These findings suggest that neuroprotection and shifts toward or away from a progenitor-like phenotype are independent activities. Inhibition of Notch in Müller glia in undamaged retinas may deplete numbers of glia capable of proliferation and transdifferentiation. We propose a model for interactions between MAPK and Notch pathways that exert independent and interdependent effects on Müller glia in a context-dependent manner (Fig. 9).

We conclude that Notch signaling influences the phenotype and function of mature Müller glia in the retina. Low levels of Notch signaling diminish the neuroprotective capacity of Müller glia, but are required to maintain the ability of Müller glia to become progenitor-like cells. In addition, we conclude that there is cross talk between Notch and MAPK pathways. FGF2 induces the expression of Notch pathway genes and Notch signaling is required for the FGF2-mediated accumulation of p38 MAPK and pCREB in Müller glia. Further, Notch signaling is essential for the proliferation-stimulating effects of FGF/MAPK in Müller glia in damaged retinas.

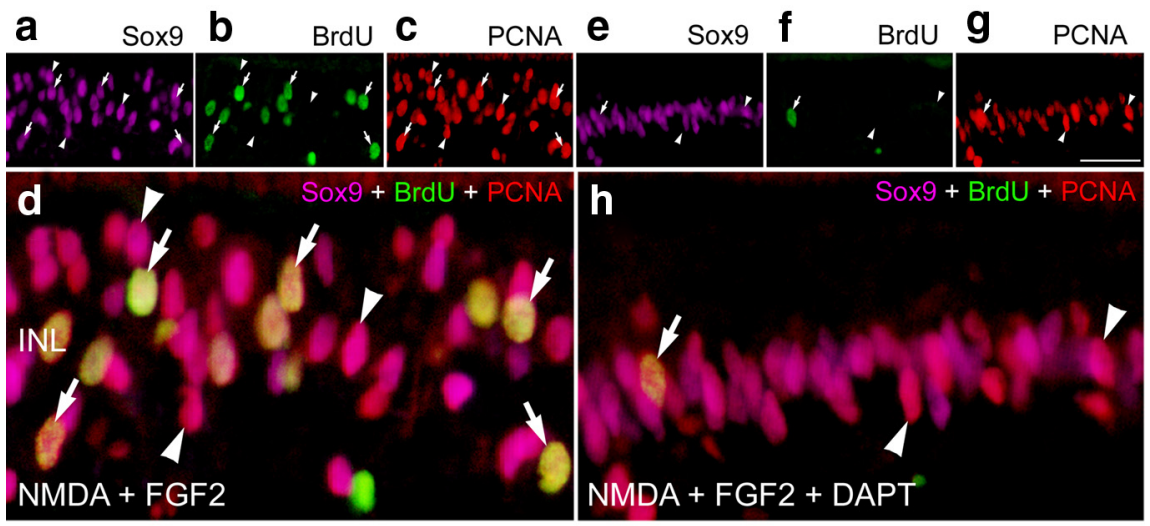

Figure 8. Inhibition of Notch signaling blocks FGF2-induced proliferation of Müller glia in NMDA-damaged retinas. Eyes were arrowheads indicate Sox9- and PCNA-double-positive cells. The histograms in $i$ represent the mean ( \pm SD) number of Sox9BrdU-, and PCNA-triple-positive cells per $15,000 \mu \mathrm{m}^{2}$ of retina. Significance of difference $\left({ }^{* *} p<0.001\right)$ was determined by using a two-tailed, unpaired Student's $t$ test. INL, Inner nuclear layer. The scale bar $(50 \mu \mathrm{m})$ in $\boldsymbol{g}$ applies to $\boldsymbol{a}-\boldsymbol{c}$ and $\boldsymbol{e} \boldsymbol{g}$.

\section{References}

Ahmad I, Zaqouras P, Artavanis-Tsakonas S (1995) Involvement of Notch-1 in mammalian retinal neurogenesis: association of Notch-1 activity with both immature and terminally differentiated cells. Mech Dev 53:73-85

Anezary L, Medina JI, Sánchez-Nogueiro J, López-Gallardo M, Prada C (2001) Shape diversity among chick retina Muller cells and their postnatal differentiation. J Comp Neurol 438:32-49.

Austin CP, Feldman DE, Ida JA Jr, Cepko CL (1995) Vertebrate retinal ganglion cells are selected from competent progenitors by the action of Notch. Development 121:3637-3650.

Badea TC, Cahill H, Ecker J, Hattar S, Nathans J (2009) Distinct roles of transcription factors brn3a and brn3b in controlling the development, morphology, and function of retinal ganglion cells. Neuron 61:852-864.

Bao ZZ, Cepko CL (1997) The expression and function of Notch pathway genes in the developing rat eye. J Neurosci 17:1425-1434.

Breunig JJ, Silbereis J, Vaccarino FM, Sestan N, Rakic P (2007) Notch regulates cell fate and dendrite morphology of newborn neurons in the postnatal dentate gyrus. Proc Natl Acad Sci U S A 104:20558-20563.

Bringmann A, Reichenbach A (2001) Role of Muller cells in retinal degenerations. Front Biosci 6:E72-E92.

Bringmann A, Pannicke T, Grosche J, Francke M, Wiedemann P, Skatchkov SN, Osborne NN, Reichenbach A (2006) Muller cells in the healthy and diseased retina. Prog Retin Eye Res 25:397-424.

Dorsky RI, Chang WS, Rapaport DH, Harris WA (1997) Regulation of neuronal diversity in the Xenopus retina by Delta signalling. Nature 385:67-70

Faux CH, Turnley AM, Epa R, Cappai R, Bartlett PF (2001) Interactions between fibroblast growth factors and Notch regulate neuronal differentiation. J Neurosci 21:5587-5596. 


\section{a Insulin/IGF1 alone}

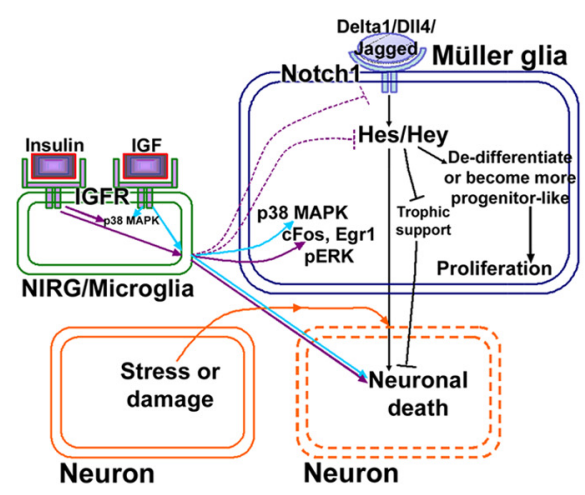

C Insulin/IGF1 and FGF2 b FGF2 alone
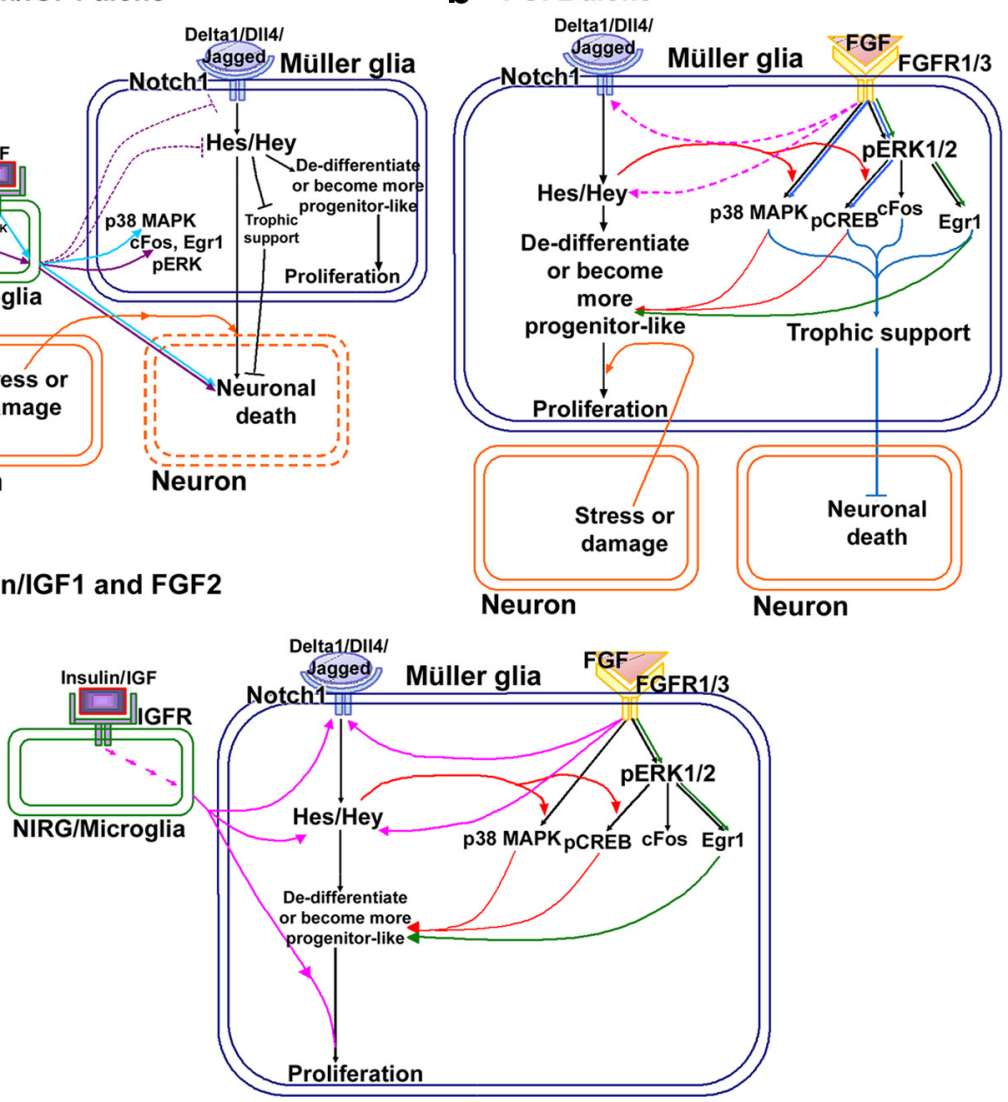

Figure 9. Model for the influence of the Notch and MAPK pathways on retinal Müller glia. This figure draws from several studies to construct putative modes of action for insulin/IGF, FGF2, and neuronal damage on Müller glia in the chick retina (Fischer et al., 2002b, 2004b, 2009a,b). $a$, Effects of insulin/IGF1 alone: insulin/IGF1 signaling via the NIRG cells and/or microglia secondarily enhances effectors of MAPK signaling, i.e., p38 MAPK (IGF1-mediated) and CFos, Egr1, and pERK (insulin-mediated) in Müller glia in addition to increasing neuronal cell death (magenta arrows). It also modestly decreases Notch and related genes (magenta dashed arrows). Low levels of Notch signaling in Müller glia inhibits the neuron-supporting functions of Müller glia, exacerbating neuronal death (black arrows) during damage (orange arrows). In addition, low levels of Notch signaling enhance dedifferentiation and progenitor-like properties (black arrows), promoting Müller glial proliferation during damage. $\boldsymbol{b}$, Effects of FGF2 alone: FGF2 has Notch-dependent and Notch-independent effects. (1) Notch-independent effects-FGF/MAPK signaling induces the accumulation of pERK1/2, p38 MAPK, pCREB, cfos, and Egr1 in Müller glia, which may stimulate the Müller glia to become neuroprotective and provide support to neurons in damaged retinas (blue arrows). pERK1/2 and Egr1 promote Müller glial dedifferentiation and proliferation in a Notch-independent manner (green arrows). (2) Notch-dependent effects—FGF/MAPK signaling induces upregulates expression of Notch and associated genes (pink dashed arrows). Low levels of Notch signaling in an undamaged or moderately damaged retina "prime" the glia to proliferate (black arrows). FGF2-mediated Müller glial proliferation requires some retinal damage and active Notch signaling, which promotes accumulation of p38 MAPK and pCREB (red arrows), which may make the glia more progenitor-like, inducing Müller glial proliferation during damage (orange arrow). c, Combined effects for three consecutive daily doses of insulin/IGF1 and FGF2: insulin/IGF1 signaling in the NIRG cells/microglia and FGF/MAPK signaling in the Müller glia together upregulate expression of Notch and its downstream effectors (pink arrows). Insulin/IGF1 signaling, FGF/MAPK signaling, and upregulated Notch signaling together induce the dedifferentiation and proliferation of Müller glia in the absence of damage.

Fischer AJ, Omar G (2005) Transitin, a nestin-related intermediate filament, is expressed by neural progenitors and can be induced in Muller glia in the chicken retina. J Comp Neurol 484:1-14.

Fischer AJ, Reh TA (2001) Muller glia are a potential source of neural regeneration in the postnatal chicken retina. Nat Neurosci 4:247-252.

Fischer AJ, Reh TA (2002) Exogenous growth factors stimulate the regeneration of ganglion cells in the chicken retina. Dev Biol 251:367-379.

Fischer AJ, Reh TA (2003) Potential of Muller glia to become neurogenic retinal progenitor cells. Glia 43:70-76.

Fischer AJ, Seltner RL, Poon J, Stell WK (1998) Immunocytochemical characterization of quisqualic acid- and N-methyl-D-aspartate-induced excitotoxicity in the retina of chicks. J Comp Neurol 393:1-15.

Fischer AJ, Morgan IG, Stell WK (1999) Colchicine causes excessive ocular growth and myopia in chicks. Vision Res 39:685-697.

Fischer AJ, Dierks BD, Reh TA (2002a) Exogenous growth factors induce the production of ganglion cells at the retinal margin. Development 129:2283-2291.

Fischer AJ, McGuire CR, Dierks BD, Reh TA (2002b) Insulin and fibroblast growth factor 2 activate a neurogenic program in Muller glia of the chicken retina. J Neurosci 22:9387-9398.

Fischer AJ, Schmidt M, Omar G, Reh TA (2004a) BMP4 and CNTF are neuroprotective and suppress damage-induced proliferation of Muller glia in the retina. Mol Cell Neurosci 27:531-542.

Fischer AJ, Omar G, Eubanks J, McGuire CR, Dierks BD, Reh TA (2004b) Different aspects of gliosis in retinal Muller glia can be induced by CNTF, insulin and FGF2 in the absence of damage. Mol Vis 10:973-986.

Fischer AJ, Ritchey ER, Scott MA, Wynne A (2008) Bullwhip neurons in the retina regulate the size and shape of the eye. Dev Biol 317:196-212.

Fischer AJ, Scott MA, Tuten W (2009a) Mitogenactivated protein kinase-signaling stimulates Muller glia to proliferate in acutely damaged chicken retina. Glia 57:166-181.

Fischer AJ, Scott MA, Ritchey ER, Sherwood P (2009b) Mitogen-activated protein kinasesignaling regulates the ability of Muller glia to proliferate and protect retinal neurons against excitotoxicity. Glia 57:1538-1552.

Fischer AJ, Scott MA, Zelinka C, Sherwood P (2010) A novel type of glial cell in the retina is stimulated by insulin-like growth factor 1 and may exacerbate damage to neurons and Müller glia. Glia, in press.

Furukawa T, Mukherjee S, Bao ZZ, Morrow EM, Cepko CL (2000) rax, Hes1, and notch1 promote the formation of Muller glia by postnatal retinal progenitor cells. Neuron 26:383-394.

Gaiano N, Fishell G (2002) The role of notch in promoting glial and neural stem cell fates. Annu Rev Neurosci 25:471-490.

Gaiano N, Nye JS, Fishell G (2000) Radial glial identity is promoted by Notch 1 signaling in the murine forebrain. Neuron 26:395-404.

Ghai K, Stanke JJ, Fischer AJ (2008) Patterning of the circumferential marginal zone of progenitors in the chicken retina. Brain Res 1192:76-89.

Ghai K, Zelinka C, Fischer AJ (2009) Serotonin released from amacrine neurons is scavenged and degraded by bipolar neurons in the retina. J Neurochem 111:1-14.

Grewal SS, York RD, Stork PJ (1999) Extracellularsignal-regulated kinase signalling in neurons. Curr Opin Neurobiol 9:544-553.

Harada T, Harada C, Kohsaka S, Wada E, Yoshida K, Ohno S, Mamada H, Tanaka K, Parada LF, Wada K (2002) Microglia-Muller glia cell interactions control neurotrophic factor production during lightinduced retinal degeneration. J Neurosci 22:9228-9236.

Harman AM, Beazley LD (1989) Generation of retinal cells in the wallaby, Setonix brachyurus (quokka). Neuroscience 28:219-232.

Hayes S, Nelson BR, Buckingham B, Reh TA (2007) Notch signaling regulates regeneration in the avian retina. Dev Biol 312:300-311.

Henrique D, Hirsinger E, Adam J, Le Roux I, Pourquié O, Ish-Horowicz D, Lewis J (1997) Maintenance of neuroepithelial progenitor cells by DeltaNotch signalling in the embryonic chick retina. Curr Biol 7:661-670.

Inman DM, Horner PJ (2007) Reactive nonproliferative gliosis predominates in a chronic mouse model of glaucoma. Glia 55:942-953.

Kageyama R, Ohtsuka T (1999) The Notch-Hes pathway in mammalian neural development. Cell Res 9:179-188.

Karl MO, Hayes S, Nelson BR, Tan K, Buckingham B, Reh TA (2008) Stim- 
ulation of neural regeneration in the mouse retina. Proc Natl Acad Sci U S A 105:19508-19513.

Kubo F, Nakagawa S (2009) Hairyl acts as a node downstream of Wnt signaling to maintain retinal stem cell-like progenitor cells in the chick ciliary marginal zone. Development 136:1823-1833.

Lamba DA, Karl MO, Reh TA (2009) Strategies for retinal repair: cell replacement and regeneration. Prog Brain Res 175:23-31.

Linser PJ, Schlosshauer B, Galileo DS, Buzzi WR, Lewis RC (1997) Late proliferation of retinal Muller cell progenitors facilitates preferential targeting with retroviral vectors in vitro. Dev Genet 20:186-196.

Liu W, Khare SL, Liang X, Peters MA, Liu X, Cepko CL, Xiang M (2000) All Brn3 genes can promote retinal ganglion cell differentiation in the chick. Development 127:3237-3247.

Louvi A, Artavanis-Tsakonas S (2006) Notch signalling in vertebrate neural development. Nat Rev Neurosci 7:93-102.

Mason HA, Rakowiecki SM, Gridley T, Fishell G (2006) Loss of notch activity in the developing central nervous system leads to increased cell death. Dev Neurosci 28:49-57.

Morgan IG (1981) Intraocular colchicine selectively destroys immature ganglion cells in chicken retina. Neurosci Lett 24:255-260.

Morgan JL, Spooner BS (1983) Immunological detection of microtubule poison-induced conformational changes in tubulin. J Biol Chem 258:13127-13133.

Nakamura K, Chiba C (2007) Evidence for Notch signaling involvement in retinal regeneration of adult newt. Brain Res 1136:28-42.

Nelson BR, Reh TA (2008) Relationship between Delta-like and proneural bHLH genes during chick retinal development. Dev Dyn 237:1565-1580.

Nelson BR, Gumuscu B, Hartman BH, Reh TA (2006) Notch activity is downregulated just prior to retinal ganglion cell differentiation. Dev Neurosci 28:128-141.

Oishi K, Kamakura S, Isazawa Y, Yoshimatsu T, Kuida K, Nakafuku M, Masuyama N, Gotoh Y (2004) Notch promotes survival of neural pre- cursor cells via mechanisms distinct from those regulating neurogenesis. Dev Biol 276:172-184.

Ota M, Ito K (2006) BMP and FGF-2 regulate neurogenin-2 expression and the differentiation of sensory neurons and glia. Dev Dyn 235:646-655.

Prada C, Puga J, Pérez-Méndez L, López R, Ramírez G (1991) Spatial and temporal patterns of neurogenesis in the chick retina. Eur J Neurosci 3:559-569.

Rapaport DH, Dorsky RI (1998) Inductive competence, its significance in retinal cell fate determination and a role for Delta-Notch signaling. Semin Cell Dev Biol 9:241-247.

Roesch K, Jadhav AP, Trimarchi JM, Stadler MB, Roska B, Sun BB, Cepko CL (2008) The transcriptome of retinal Muller glial cells. J Comp Neurol 509:225-238.

Saravanamuthu SS, Gao CY, Zelenka PS (2009) Notch signaling is required for lateral induction of Jagged1 during FGF-induced lens fiber differentiation. Dev Biol 332:166-176.

Sullivan SA, Barthel LK, Largent BL, Raymond PA (1997) A goldfish Notch-3 homologue is expressed in neurogenic regions of embryonic, adult, and regenerating brain and retina. Dev Genet 20:208-223.

Valsecchi C, Ghezzi C, Ballabio A, Rugarli EI (1997) JAGGED2: a putative Notch ligand expressed in the apical ectodermal ridge and in sites of epithelial-mesenchymal interactions. Mech Dev 69:203-207.

Vetter ML, Moore KB (2001) Becoming glial in the neural retina. Dev Dyn 221:146-153.

Wahl MB, Deng C, Lewandoski M, Pourquié O (2007) FGF signaling acts upstream of the NOTCH and WNT signaling pathways to control segmentation clock oscillations in mouse somitogenesis. Development 134: 4033-4041.

Yurco P, Cameron DA (2005) Responses of Muller glia to retinal injury in adult zebrafish. Vision Res 45:991-1002.

Yurco P, Cameron DA (2007) Cellular correlates of proneural and Notchdelta gene expression in the regenerating zebrafish retina. Vis Neurosci 24:437-443. 